\title{
Hermite-Hadamard Type Fuzzy Inequalities based on s-Convex Function in the Second Sense
}

\author{
Lanping Li \\ School of Mathematics and Statistics, Hunan University of Finance and Economics, Changsha, China
}

Email address:

Lilanping1981@163.com

To cite this article:

Lanping Li. Hermite-Hadamard Type Fuzzy Inequalities based on s-Convex Function in the Second Sense. Mathematics Letters. Vol. 3, No. 6, 2017, pp. 77-82. doi: 10.11648/j.ml.20170306.14

Received: October 27, 2017; Accepted: November 14, 2017; Published: December 5, 2017

\begin{abstract}
Integral inequalities have important applications in propability and engineering field. Sugeno integral is an important fuzzy integral in fuzzy theory, which has many applications in various fields. The object of this paper is to develop some new integral inequalities for Sugeno integral. Based on classical Hermite-Hadamard type inequality, this paper intends to extend it for the Sugeno integral. Some new Hermite-Hadamard type inequalities are derived for Sugeno integral based on sconvex function in the second sense. An example is used to illustrate the effectiveness of the new inequalities.
\end{abstract}

Keywords: Fuzzy Integral, Sugeno Integral, Herimite-Hadamard Inequality, s-Convex Function

\section{Introduction}

In some practical application problems, the data information sometimes cannot be precisely expressed due to human errors or the limitation of decision maker's knowledge or other reasons. For instance, the temperature in a room cannot be measured exactly because of the fluctuation [1]. Zadeh first introduce the concept of fuzzy set. Since then fuzzy set has received great attention and got lots of applications in various fields [2-5]. Many extensions of Zadeh's fuzzy set, such as vague set, intuitionistic fuzzy, hesitant fuzzy set, etc., have been proposed and been utilized management decision and engineering science problems [612]. Fuzzy integral, first introduced by Sugeno in 1974, is an important analytical tool to measure uncertain information [13-18].

Hermite-Hadamard inequalities are important integral inequality for convex functions. Many results are derived for Hermite-Hadamard inequalities based on various type convex functions. For example, Wang et al. [17] established some new Hermite-Hadamard type inequalities involving Riemann-Liouville fractional integrals via s-convex functions in the second sense. Sarikaya [18] derived established Hermite-Hadamard type inequalities for the class of function whose second derivatives are s-convex in the second sense. Latif [19] established several new inequalities of the Hermite-Hadamard type for functions whose derivatives are s-convex in the second sense in the absolute value. Hosseini et al. [20] derived several versions of Hermite-Hadamard type inequality for pseudo-fractional integrals.

The study of inequalities for Sugeno integral, which was initiated by Roman-Flores et al. [21], is most popular. From above discussion, we see that most of extended HermiteHadamard inequalities are established for definite integral, but the research based on fuzzy integral is still rare [22-26]. s-convex function in the second sense is a kind of important convex function, and ordinary convex function is its special case [27]. Under definite integral, many authors are interested in building inequality for this function. In this paper, we will extend the Hermite-Hadamard type inequality for s-convex functions in the second sense for Sugneo integral. The organization of this article is as follows: Second 2 will recall the definitions and properties of Sugeno integral and convex function. Section 3 will establish some new Hermite-Hadamard type inequalities for s-convex function in the second sense based on Sugeno integral. Finally, conclusions are provided in Section 4.

\section{Preliminaries}

In this section, some concepts and properties of Sugeno integral and s-convex function in the second sense are introduced. In the follow we always denote by $R$ the set of real numbers. Supposed that $X$ is a nonempty set and 
$R_{+}=[0, \infty), \Sigma$ is a $\sigma$-algebra of subsets of $X$.

Definition 1 [23]. We call a mapping $\mu: \Sigma \rightarrow R_{+}$a nonadditive measure, if it is a non-negative set function, and satisfies the following properties:

(i) $\mu(\Phi)=0$.

(ii) $A, B \in \Sigma$ and $A \subset B \Rightarrow \mu(A) \leq \mu(B)$.

(iii) For all $n \geq 1, A_{n} \in \Sigma$ and $A_{1} \subset A_{2} \subset \cdots, \Rightarrow$ $\mu\left(\bigcup_{n=1}^{\infty} A_{n}\right)=\lim _{n \rightarrow \infty} \mu\left(A_{n}\right)$.

(iv) For all $n \geq 1, A_{n} \in \Sigma, A_{1} \supset A_{2} \supset \cdots$ and $\mu\left(A_{1}\right)<+\infty$, $\Rightarrow \mu\left(\bigcap_{n=1}^{\infty} A_{n}\right)=\lim _{n \rightarrow \infty} \mu\left(A_{n}\right)$.

The triple $(X, \Sigma, \mu)$ is called a fuzzy measure space. For any $\alpha \geq 0$, we denote

$$
F_{\alpha}=\{x \mid x \in X, f(x) \geq \alpha\}=\{f \geq \alpha\} .
$$

Then it is easily proved that if $\alpha \leq \beta$ then $F_{\beta} \subset F_{\alpha}$.

Definition $2[1,28]$. Let $(X, \Sigma, \mu)$ be a fuzzy measure space, $f: X \rightarrow R_{+}$be a non-negative measurable function, $A \in \Sigma$. The Sugeno integral of function $f$ on set $A$ is defined as

$$
(S) \int_{A} f d \mu=\underset{\alpha \geq 0}{\vee}\left[\alpha \wedge \mu\left(A \cap F_{\alpha}\right)\right]
$$

In particular, if $A=X$, then

$$
\text { (S) } \int_{X} f d \mu=\underset{\alpha \geq 0}{\vee}\left[\alpha \wedge \mu\left(F_{\alpha}\right)\right]
$$

Here $\vee$ and $\wedge$ are the operations sup and inf on $R_{+}=[0, \infty)$, respectively.

Proposition 1 [28]. Let $(X, \Sigma, \mu)$ be a fuzzy measure space, $A, B \in \Sigma . f$ and $g$ are non-negative measurable functions. Then

(i) $(S) \int_{A} f d \mu \leq \mu(A)$

(ii) (S) $\int_{A} k d \mu \leq k \wedge \mu(A), \forall k \in R_{+}$,

(iii) If $f \leq g$ on the set $A$, then

$$
(S) \int_{A} f d \mu \leq(S) \int_{A} g d \mu .
$$

(iv) If $A, B \in \Sigma, A \subset B$, then

$$
(S) \int_{A} f d \mu \leq(S) \int_{B} f d \mu .
$$

(v) If $\mu(A \cap\{f \geq \alpha\}) \geq \alpha$, then

$$
(S) \int_{A} f d \mu \geq \alpha
$$

(vi) If $\mu(A \cap\{f \geq \alpha\}) \leq \alpha$, then $(S) \int_{A} f d \mu \leq \alpha$.

(vii) $(S) \int_{A} f d \mu<\alpha \Leftrightarrow$

There exists $\gamma<\alpha$, s.t. $\mu(A \cap\{f \geq \gamma\})<\alpha$.

(IX) $(S) \int_{A} f d \mu>\alpha \Leftrightarrow$

There exists $\gamma>\alpha$, s.t. $\mu(A \cap\{f \geq \gamma\})>\alpha$.

(X) $\mu(A)<+\infty$, then

(S) $\int_{A} f d \mu \geq \alpha \Leftrightarrow \mu(A \cap\{f \geq \alpha\}) \geq \alpha$.

Remark 1. Let the distribution associated to $f$ on $A$ is $F(\alpha)=\mu(A \cap\{f \geq \alpha\})$, then according to the properties (V) and (VI) of Proposition 1, we have that

$$
F(\alpha)=\alpha \Rightarrow(S) \int_{A} f d \mu=\alpha
$$

Then from a numerical point of view, the Sugeno integral (1) can be solvedas the solution of the equation $F(\alpha)=\alpha$.

Definition3 [27]. Let $s \in(0,1]$ be a real number. A function $f: I \subseteq R_{+} \rightarrow R$ is said to be s-convex in the second sense if

$$
f(\lambda x+(1-\lambda) y) \leq \lambda^{s} f(x)+(1-\lambda)^{s} f(y)
$$

holds for all $(x, y) \in I$ and $\lambda \in[0,1]$.

Remark 2. If $\mathrm{s}=1$, then one can obtain the definition of ordinary convex function. Denote by $K_{s}^{2}$ the set of all sconvex functions in the second sense.

Lemma 1 [29]. Let $x \geq 0, y \geq 0$, then the inequality

$$
(x+y)^{\theta} \leq x^{\theta}+y^{\theta}
$$

holds for $\theta \in(0,1]$.

Lemma 2 [29]. Let $x \in[0,1]$, then the inequality

$$
(1-x)^{s} \leq 2^{1-s}-x^{s}
$$

holds for $s \in(0,1]$.

\section{Hermite-Hadamard Type Inequalities for Sugeno IntegralBased on s-Convex in the Second Sense}

Hermite-Hadamard Type inequality provides estimates ofthe mean value of a nonnegative and convex function $f:[a, b] \rightarrow R$ with the following inequality ([30-33])

$$
f\left(\frac{a+b}{2}\right) \leq \frac{1}{b-a} \int_{a}^{b} f(x) d x \leq \frac{f(a)+f(b)}{2}
$$

Unfortunately, Example 1will show that HermiteHadamard typeinequality for Sugeno integral based on sconvex functions in the second senseis not valid. 
Example 1. Consider the universe set $X=[0,1]$ and let $\mu$ be the Lebesgue measure on $X$. If we take the function $f(x)=\frac{x^{2}}{4}$, then by reference [27], we can easily know that $f(x) \in K_{s}^{2}$. Calculate the Sugeno integral $(S) \int_{0}^{1} f d \mu$ by Remark 1, we get

$$
(S) \int_{0}^{1} f d \mu=3-2 \sqrt{2} \approx 0.172
$$

On the other hand,

$$
\frac{f(0)+f(1)}{2}=0.125
$$

This proves that the Hermite-Hadamard type inequality is not satisfiedfor Sugeno integral based on s-convex functions in the second sense.

In this section, we will derived some new HermiteHadamard type inequalities for the Sugeno integral based on s-convex functions in the second sense.

Theorem 1. Let $s \in(0,1]$ and $f:[0,1] \rightarrow[0, \infty)$ is a sconvex function in the second sense, such that $f(1)>f(0)$ and let $\mu$ be the Lebesgue measure on $R$, then

$$
(S) \int_{a}^{b} f d \mu \leq \min \{\beta, 1\}
$$

where $\beta$ is the positive real solution of the equation

$$
1-\left(\frac{\beta-2^{1-s} f(0)}{f(1)-f(0)}\right)^{1 / s}=\beta
$$

Proof. As $f(x) \in K_{s}^{2}$ for $x \in[0,1]$, we have

$$
\begin{aligned}
f(x) & =f((1-x) \cdot 0+x \cdot 1) \\
& \leq(1-x)^{s} f(0)+x^{s} f(1)
\end{aligned}
$$

According to Lemma 2, we have

$$
f(x) \leq 2^{1-s} f(0)+x^{s}(f(1)-f(0))=g(x)
$$

Thenby (iii) of Proposition 1 and Definition 2, we have

$$
\begin{gathered}
(S) \int_{0}^{1} f(x) d \mu \leq(S) \int_{0}^{1} g(x) d \mu, \\
(S) \int_{0}^{1} g(x) d \mu=\underset{\beta \geq 0}{\vee}[\beta \wedge \mu([0,1] \cap\{g \geq \beta\})]
\end{gathered}
$$

In order to calculate the integral $(S) \int_{0}^{1} g(x) d \mu$, we consider the distribution function $F$ associated to $g(x)$ on $[0,1]$ which is given by

$$
F(\beta)=\mu([0,1] \cap\{g \geq \beta\})
$$

That is

$$
F(\beta)=\mu\left([0,1] \cap\left\{x \mid 2^{1-s} f(0)+x^{s}(f(1)-f(0)) \geq \beta\right\}\right)
$$

When $f(1)>f(0)$, then

$$
\begin{aligned}
F(\beta) & =\mu\left([0,1] \cap\left\{x \mid x \geq\left(\frac{\beta-2^{1-s} f(0)}{f(1)-f(0)}\right)^{1 / s}\right\}\right) \\
& =1-\left(\frac{\beta-2^{1-s} f(0)}{f(1)-f(0)}\right)^{1 / s}
\end{aligned}
$$

Let $F(\beta)=\beta$, then

$$
1-\left(\frac{\beta-2^{1-s} f(0)}{f(1)-f(0)}\right)^{1 / s}=\beta
$$

By (i) of Proposition 1 and (14), we have

$$
(S) \int_{0}^{1} f(x) d \mu \leq(S) \int_{0}^{1} g(x) d \mu=\min \{\beta, 1\}
$$

Then we complete the proof.

Theorem 2. Let $s \in(0,1]$ and $f:[0,1] \rightarrow[0, \infty)$ is a sconvex function in the second sense, such that $f(1)<f(0)$ and let $\mu$ be the Lebesgue measure on $R$, then

$$
(S) \int_{0}^{1} f d \mu \leq \min \{\beta, 1\}
$$

where $\beta$ is the positive real solution of the equation

$$
\left(\frac{\beta-2^{1-s} f(0)}{f(1)-f(0)}\right)^{1 / s}=\beta
$$

Proof. Similarly to the proof of Theorem 1, we consider the function

$$
g(x)=2^{1-s} f(0)+x^{s}(f(1)-f(0))
$$

In this case, the distribution function $F$ associated to $g(x)$ on $[0,1]$ which is given by

$$
F(\beta)=\mu([0,1] \cap\{g \geq \beta\})
$$

That is

$$
\begin{aligned}
F(\beta) & =\mu([0,1 \cap\{g \geq \beta\}) \\
& =\mu\left([0,1] \cap\left\{x \mid 2^{1-s} f(0)+x^{s}(f(1)-f(0)) \geq \beta\right\}\right)
\end{aligned}
$$

When $f(1)<f(0)$, then 


$$
\begin{aligned}
& F(\beta)=\mu\left([0,1] \cap\left\{x \mid x \leq\left(\frac{\beta-2^{1-s} f(0)}{f(1)-f(0)}\right)^{1 / s}\right\}\right) \\
& =\left(\frac{\beta-2^{1-s} f(0)}{f(1)-f(0)}\right)^{1 / s}
\end{aligned}
$$

Let $F(\beta)=\beta$, then

$$
\left(\frac{\beta-2^{1-s} f(0)}{f(1)-f(0)}\right)^{1 / s}=\beta
$$

By (i) of Proposition 1 and (18), we obtained

$$
(S) \int_{0}^{1} f(x) d \mu \leq(S) \int_{0}^{1} g(x) d \mu=\min \{\beta, 1\} .
$$

This completes the proof.

Remark 3 . In the case $f(1)=f(0)$, then function $g(x)$ of Theorems 1 and 2 is

$$
g(x)=2^{1-s} f(0) .
$$

According to (ii) of Proposition 1, we have

$$
(S) \int_{0}^{1} f(x) d \mu \leq(S) \int_{0}^{1} g(x) d \mu=\min \left\{2^{1-s} f(0), 1\right\}
$$

Now, we will prove the general case of Theorem 1 and Theorem 2.

Theorem 3. Let $s \in(0,1]$ and $f:[a, b] \rightarrow[0, \infty)$ is a sconvex function in the second sense, such that $f(b)>f(a)$ and let $\mu$ be the Lebesgue measure on $R$, then

$$
(S) \int_{a}^{b} f d \mu \leq \min \{\beta, b-a\}
$$

where $\beta$ is the positive real solution of the equation

$$
(b-a)\left[1-\left(\frac{\beta-2^{1-s} f(a)}{f(b)-f(a)}\right)^{1 / s}\right]=\beta
$$

Proof. As $f(x) \in K_{s}^{2}$ for $x \in[a, b]$, we have

$$
\begin{aligned}
f(x) & =f\left(\left(1-\frac{x-m a}{b-m a}\right) a+\left(\frac{x-m a}{b-m a}\right) b\right) \\
& \leq\left(1-\frac{x-m a}{b-m a}\right)^{s} f(a)+\left(\frac{x-m a}{b-m a}\right)^{s} f(b)
\end{aligned}
$$

According to Lemma 2, we have

$$
\left(1-\frac{x-a}{b-a}\right)^{s} \leq 2^{1-s}-\left(\frac{x-a}{b-a}\right)^{s}
$$

$$
f(x) \leq g(x)
$$

Where

$$
g(x)=2^{1-s} f(a)+\left(\frac{x-m a}{b-m a}\right)^{s}(f(b)-f(a))
$$

Thenby (iii) of Proposition 1 and Definition 2, we have

$$
(S) \int_{a}^{b} f(x) d \mu \leq(S) \int_{a}^{b} g(x) d \mu
$$

$$
(S) \int_{a}^{b} g(x) d \mu=\underset{\beta \geq 0}{\vee}[\beta \wedge \mu([a, b] \cap\{g \geq \beta\})]
$$

In order to calculate the integral $(S) \int_{a}^{b} g(x) d \mu$, we consider the distribution function $F$ associated to $g(x)$ on $[a, b]$ which is given by

$$
F(\beta)=\mu([a, b] \cap\{g \geq \beta\})
$$

That is

$$
F(\beta)=\mu\left([a, b] \cap\left\{x \mid 2^{1-s} f(a)+\left(\frac{x-m a}{b-m a}\right)^{s}(f(b)-f(a)) \geq \beta\right\}\right)
$$

When $f(b)>f(a)$, then

$$
\begin{aligned}
F(\beta) & =\mu\left([a, b] \cap\left\{x \mid x \geq m a+(b-m a)\left(\frac{\beta-2^{1-s} f(a)}{f(b)-f(a)}\right)^{1 / s}\right\}\right) \\
& =(b-m a)\left[1-\left(\frac{\beta-2^{1-s} f(a)}{f(b)-f(a)}\right)^{1 / s}\right]
\end{aligned}
$$

Let $F(\beta)=\beta$, then

$$
(b-m a)\left[1-\left(\frac{\beta-2^{1-s} f(a)}{f(b)-f(a)}\right)^{1 / s}\right]=\beta
$$

By (i) of Proposition 1 and (27), we obtained

$$
(S) \int_{a}^{b} f(x) d \mu \leq(S) \int_{a}^{b} g(x) d \mu=\min \{\beta, b-a\}
$$

Then we complete the proof.

Theorem 4. Let $s \in(0,1]$ and $f:[a, b] \rightarrow[0, \infty)$ is a sconvex function in the second sense, such that $f(b)<m f(a)$ and let $\mu$ be the Lebesgue measure on $R$, then

$$
\text { (S) } \int_{a}^{b} f d \mu \leq \min \{\beta, b-a\}
$$

where $\beta$ is the positive real solution of the equation

Then 


$$
(b-m a)\left(\frac{\beta-2^{1-s} f(a)}{f(b)-f(a)}\right)^{1 / s}=\beta
$$

Proof. Similarly to the proof of Theorem 1, we consider the function

$$
g(x)=2^{1-s} f(a)+\left(\frac{x-m a}{b-m a}\right)^{s}(f(b)-f(a))
$$

In this case, the distribution function $F$ associated to $g(x)$ on $[a, b]$ which is given by

$$
F(\beta)=\mu([a, b] \cap\{g \geq \beta\})
$$

That is

$$
\begin{aligned}
F(\beta) & =\mu([a, b] \cap\{g \geq \beta\}) \\
& =\mu\left([a, b] \cap\left\{x \mid 2^{1-s} f(a)+\left(\frac{x-m a}{b-m a}\right)^{s}(f(b)-f(a)) \geq \beta\right\}\right)
\end{aligned}
$$

When $f(b)<f(a)$, then

$$
\begin{aligned}
F(\beta) & =\mu\left([a, b] \cap\left\{x \mid x \leq m a+(b-m a)\left(\frac{\beta-2^{1-s} f(a)}{f(b)-f(a)}\right)^{1 / s}\right\}\right) \\
& =(b-m a)\left(\frac{\beta-2^{1-s} f(a)}{f(b)-f(a)}\right)^{1 / s}
\end{aligned}
$$

Let $F(\beta)=\beta$, then

$$
(b-m a)\left(\frac{\beta-2^{1-s} f(a)}{f(b)-f(a)}\right)^{1 / s}=\beta
$$

By (i) of Proposition 1 and (31), we obtained

$$
(S) \int_{a}^{b} f(x) d \mu \leq(S) \int_{a}^{b} g(x) d \mu=\min \{\beta, b-a\} .
$$

This completes the proof.

To show the valid and effectiveness of the above established Hermite-Hadamard type fuzzy inequalities, we see the following example.

Example2. Consider $X=[0,1]$ and let $\mu$ be the Lebesgue measure on $X$. If we take the function $f(x)=\sqrt{x}$, then by Remark 3, we know that $f(x) \in K_{s}^{2}$ for $s \in\left(0, \frac{1}{2}\right]$.

Here we let $s=\frac{1}{2}$, then $f(1)>f(0)$. By Theorem 1, through solving the equation

$$
1-\left(\frac{\beta-\sqrt{2} f(0)}{f(1)-f(0)}\right)^{2}=\beta
$$

we get $\beta=\frac{\sqrt{5}-1}{2}=0.618$. Then

$$
\text { (S) } \int_{0}^{1} f d \mu \leq \min \{\beta, 1-0\}=0.618 \text {. }
$$

Straightforward calculation shows that

$$
\text { (S) } \int_{0}^{1} f d \mu=0.618 \text {. }
$$

This also implies the inequality can get a well estimate of Sugeno integral $(S) \int_{0}^{1} f d \mu$.

\section{Conclusions}

Sugeno integral is an important fuzzy integral in fuzzy theory, which has many applications in management decision and engineering fields. Integral inequalities are an important tool for estimating the values of integrals. Hermite-Hadamard type inequalities provide estimates of the mean value of a nonnegative and ordinary convex function, and thus received great attention in definite integral.

The main contribution of this paper is to develop HermiteHadamard type inequalities for Sugeno integral. This paper established an upper approximation for the Sugeno integral of s-convex functions in the second sense.

In the future study, we will study the other properties and inequalities about s-convex functions in the second sense for some other fuzzy integrals, such as Choquet integral, seminormed fuzzy integral, etc.

\section{Acknowledgements}

The author is grateful to the reviewers for a very careful reading of the manuscript and the suggestions that lead to the improvement of the paper. This study is partially supported by 933rd items of Education Reform Project in Hunan Province in 2016.

\section{References}

[1] Wu H C. Fuzzy Bayesian estimation on lifetime data [J]. Computational Statistics, 2004, 19(4): 613.

[2] Dubois D, Prade H, Esteva F, et al. Fuzzy set modelling in case-based reasoning [J]. International Journal of Intelligent Systems, 2015, 13(4): 345-373.

[3] Setz S, Semling M, Mülhaupt R. Fuzzy set approach for fitting a continuous response surface in adhesion formulation[J]. Journal of Chemometrics, 2015, 11(5): 403-418.

[4] Dalman H, Güzel N, Sivri M. A fuzzy set-based approach to multi-objective multi-item solid transportation problem under uncertainty [J]. International Journal of Fuzzy Systems, 2016, 18(4): 716-729.

[5] Wang W, Liu X. Fuzzy forecasting based on automatic clustering and axiomatic fuzzy set classification [J]. Information Sciences, 2015, 294(294): 78-94. 
[6] Liu C, Zuo X. A study on dynamic evaluation of urban integrated natural disaster risk based on vague set and information axiom [J]. Natural Hazards, 2015, 78(3): 15011516.

[7] Wang L, Yu L, Qiao N, et al. Analysis and Simulation of Geomagnetic Map Suitability Based on Vague Set [J]. Journal of Navigation, 2016, 69(5): 1114-1124.

[8] Chen T Y. The inclusion-based TOPSIS method with intervalvalued intuitionistic fuzzy sets for multiple criteria group decision making $[\mathrm{J}]$. Applied Soft Computing, 2015, 26: 5773.

[9] Dong J, Wan S. A new method for multi-attribute group decision making with triangular intuitionistic fuzzy numbers [J]. Kybernetes, 2016, 45(1): 158-180.

[10] Zhang $\mathrm{X}, \mathrm{Xu} \mathrm{Z}$. Soft computing based on maximizing consensus and fuzzy TOPSIS approach to interval-valued intuitionistic fuzzy group decision making [J]. Applied Soft Computing, 2015, 26(26): 42-56.

[11] Meng F, Chen X. Correlation coefficients of hesitant fuzzy sets and their application based on fuzzy measures [J]. Cognitive Computation, 2015, 7(4): 445-463.

[12] Meng F, Wang C, Chen X, et al. Correlation coefficients of interval-valued hesitant fuzzy sets and their application based on the Shapley function [J]. International Journal of Intelligent Systems, 2016, 31(1): 17-43.

[13] Abbaszadeh S, Gordji M E, Pap E, et al. Jensen-type inequalities for Sugeno integral [J]. Information Sciences, 2016, 376: 148-157.

[14] Zhang X, Zheng Y. Linguistic quantifiers modeled by intervalvalued intuitionistic Sugeno integrals [J]. Journal of Intelligent \& Fuzzy Systems, 2015, 29(2): 583-592.

[15] Yager R R, Alajlan N. Sugeno integral with possibilistic inputs with application to multi-criteria decision making $[\mathrm{J}]$. International Journal of Intelligent Systems, 2016, 31(8):813826.

[16] Pap E. Theory and applications of non-additive measures and corresponding integrals $[\mathrm{J}]$. Lecture Notes in Computer Science, 2015, 8234: 1-10.

[17] Wang J, Li X, Zhou Y. Hermite-Hadamard inequalities involving Riemann-Liouville fractional integrals via s-convex functions and applications to special means [J]. Filomat, 2016, 30(5): 1143-1150.

[18] Sarikaya M Z, Kiris M E. Some new inequalities of hermitehadamard type for s-convex functions [J]. Miskolc Mathematical Notes, 2015, 16(1): 491-501.

[19] Latif M A. On Some new inequalities of Hermite-Hadamard type for functions whose derivatives are s-convex in the decond sense in the absolute value $[\mathrm{J}]$. Ukrainian Mathematical Journal, 2015, 67(10): 1-20.

[20] Hosseini M, Babakhani A, Agahi H, et al. On pseudofractional integral inequalities related to Hermite-Hadamard type [J]. Soft Computing, 2016, 20(7): 2521-2529.

[21] Román-Flores H, Flores-Franulic A, Chalco-Cano Y. The fuzzy integral for monotone functions. Applied Mathematics \& Computation, 2007, 185(1): 492-498.

[22] Turhan S, Bekar N O, Akdemir H G. Hermite-Hadamard Type Inequality for Log-convex Functions via Sugeno Integrals[J]. Mathematics, 2015.

[23] Caballero J, Sadarangani K. Hermite-Hadamard inequality for fuzzy integrals[J]. Applied Mathematics \& Computation, 2009, 215(6): 2134-2138.

[24] Li D Q, Song X Q, Yue T. Hermite-Hadamard type inequality for Sugeno integrals[J]. Applied Mathematics \& Computation, 2014, 237(3): 632-638.

[25] Abbaszadeh S, Eshaghi M. A Hadamard-type inequality for fuzzy integrals based on r-convex functions [J]. Soft Computing, 2016, 20(8), 3117-3124.

[26] Latif M A, Irshad W, Mushtaq M. HermiteHadamardtypeinequalitiesfor $\mathrm{m}$-convex and (a,m)-convex functions for fuzzy integrals [J]. Journal of Computational Analysis \& Applications, 2018, 24(3): 497-506.

[27] Hudzik H, Maligranda L. Some remarks on s -convex functions [J]. Aequationes Mathematicae, 1994, 48(1): 100111.

[28] Z. Wang, G. Klir, Fuzzy Measure Theory [M], Plenum, New York, 1992.

[29] Ren H, Wang G, Luo L. Sandor type fuzzy inequality based on the $(\mathrm{s}, \mathrm{m})$-convex function in the second sense [J]. Symmetry, 2017, 9(9): 181-190.

[30] Guessab A, Schmeisser G. Sharp integral inequalities of the Hermite-Hadamard Type [J]. Journal of Approximation Theory, 2002, 115(2): 260-288.

[31] Wang W, Iscan I, Zhou H. Fractional integral inequalities of Hermite-Hadamard type for $\mathrm{m}-\mathrm{HH}$ convex functions with applications [J]. Advanced Studies in Contemporary Mathematics, 2016, 26(3): 501-512.

[32] Latif M A, Dragomir S S. New integral inequalities of hermite-hadamard type for n-times differentiable slogarithmically convex functions with applications [J]. Miskolc Mathematical Notes, 2015, 16(1): 339-342.

[33] Wang S H, Shi X T. Hermite-Hadamard type inequalities for n-time differentiable and GA-convex functions with applications to means [J]. 2016, 4(1): 15-22. 